\title{
Gauss Lemma and Law of Quadratic Reciprocity
}

\author{
Li Yan \\ Qingdao University of Science \\ and Technology \\ China
}

\author{
Xiquan Liang \\ Qingdao University of Science \\ and Technology \\ China
}

\author{
Junjie Zhao \\ Qingdao University of Science \\ and Technology \\ China
}

\begin{abstract}
Summary. In this paper, we defined the quadratic residue and proved its fundamental properties on the base of some useful theorems. Then we defined the Legendre symbol and proved its useful theorems [14], [12]. Finally, Gauss Lemma and Law of Quadratic Reciprocity are proven.
\end{abstract}

MML identifier: INT_5, version: $\underline{7.8 .05 \quad 4.89 .993}$

The papers [20], [10], [9], [11], [4], [1], [2], [17], [8], [19], [7], [16], [13], [21], [22], $[5],[18],[3],[15],[6]$, and [23] provide the terminology and notation for this paper.

For simplicity, we adopt the following convention: $i, i_{1}, i_{2}, i_{3}, j, a, b, x$ denote integers, $d, e, n$ denote natural numbers, $f, f^{\prime}$ denote finite sequences of elements of $\mathbb{Z}, g, g_{1}, g_{2}$ denote finite sequences of elements of $\mathbb{R}$, and $p$ denotes a prime number.

We now state two propositions:

(1) If $i_{1} \mid i_{2}$ and $i_{1} \mid i_{3}$, then $i_{1} \mid i_{2}-i_{3}$.

(2) If $i \mid a$ and $i \mid a-b$, then $i \mid b$.

Let us consider $f$. The functor $\mathcal{P}_{\mathbb{Z}}(f)$ yields a function from $\mathbb{Z}$ into $\mathbb{Z}$ and is defined by the condition (Def. 1). 
(Def. 1) Let $x$ be an element of $\mathbb{Z}$. Then there exists a finite sequence $f^{\prime}$ of elements of $\mathbb{Z}$ such that len $f^{\prime}=\operatorname{len} f$ and for every $d$ such that $d \in \operatorname{dom} f^{\prime}$ holds $f^{\prime}(d)=f(d) \cdot x^{d-^{\prime} 1}$ and $\left(\mathcal{P}_{\mathbb{Z}}(f)\right)(x)=\sum f^{\prime}$.

Let $f$ be a finite sequence of elements of $\mathbb{Z}$ and let $x$ be an integer. Observe that $\left(\mathcal{P}_{\mathbb{Z}}(f)\right)(x)$ is integer.

We now state two propositions:

(3) If len $f=1$, then $\mathcal{P}_{\mathbb{Z}}(f)=\mathbb{Z} \longmapsto f(1)$.

(4) If len $f=1$, then for every element $x$ of $\mathbb{Z}$ holds $\left(\mathcal{P}_{\mathbb{Z}}(f)\right)(x)=f(1)$.

In the sequel $f^{\prime}$ denotes a finite sequence of elements of $\mathbb{R}$.

Next we state three propositions:

(5) Let given $g, g_{1}, g_{2}$. Suppose len $g=n+1$ and len $g_{1}=\operatorname{len} g$ and len $g_{2}=$ len $g$ and for every $d$ such that $d \in \operatorname{dom} g$ holds $g(d)=g_{1}(d)-g_{2}(d)$. Then there exists $f^{\prime}$ such that len $f^{\prime}=\operatorname{len} g-1$ and for every $d$ such that $d \in$ $\operatorname{dom} f^{\prime}$ holds $f^{\prime}(d)=g_{1}(d)-g_{2}(d+1)$ and $\sum g=\left(\left(\sum f^{\prime}\right)+g_{1}(n+1)\right)-g_{2}(1)$.

(6) Suppose len $f=n+2$. Let $a$ be an integer. Then there exists a finite sequence $f^{\prime}$ of elements of $\mathbb{Z}$ and there exists an integer $r$ such that len $f^{\prime}=$ $n+1$ and for every element $x$ of $\mathbb{Z}$ holds $\left(\mathcal{P}_{\mathbb{Z}}(f)\right)(x)=(x-a) \cdot\left(\mathcal{P}_{\mathbb{Z}}\left(f^{\prime}\right)\right)(x)+r$ and $f(n+2)=f^{\prime}(n+1)$.

(7) If $p \mid i \cdot j$, then $p \mid i$ or $p \mid j$.

In the sequel $f^{\prime}, g$ are finite sequences of elements of $\mathbb{Z}$.

The following proposition is true

(8) Let given $f$. Suppose len $f=n+1$ and $p>2$ and $p \nmid f(n+1)$. Let given $f^{\prime}$. Suppose for every $d$ such that $d \in \operatorname{dom} f^{\prime}$ holds $\left(\mathcal{P}_{\mathbb{Z}}(f)\right)\left(f^{\prime}(d)\right) \bmod p=$ 0 and for all $d, e$ such that $d, e \in \operatorname{dom} f^{\prime}$ and $d \neq e$ holds $f^{\prime}(d) \not \equiv$ $f^{\prime}(e)(\bmod p)$. Then len $f^{\prime} \leq n$.

Let $a$ be an integer and let $m$ be a natural number. We say that $a$ is quadratic residue $\bmod m$ if and only if:

(Def. 2) There exists an integer $x$ such that $\left(x^{2}-a\right) \bmod m=0$.

In the sequel $b, m$ denote natural numbers.

We now state four propositions:

(9) If $a \operatorname{gcd} m=1$, then $a^{2}$ is quadratic residue $\bmod m$.

(10) 1 is quadratic residue mod 2.

(11) If $i \operatorname{gcd} m=1$ and $i$ is quadratic residue $\bmod m$ and $i \equiv j(\bmod m)$, then $j$ is quadratic residue $\bmod m$.

(12) If $i \mid j$, then $i \operatorname{gcd} j=|i|$.

Let $k$ be an integer and let $a$ be a natural number. One can verify that $k^{a}$ is integer.

One can prove the following propositions: 
(13) For all integers $i, j, m$ such that $i \bmod m=j \bmod m$ holds $i^{n} \bmod m=$ $j^{n} \bmod m$.

(14) If $a \operatorname{gcd} p=1$ and $\left(x^{2}-a\right) \bmod p=0$, then $x$ and $p$ are relative prime.

(15) Suppose $p>2$ and $a \operatorname{gcd} p=1$ and $a$ is quadratic residue mod $p$. Then there exist integers $x, y$ such that $\left(x^{2}-a\right) \bmod p=0$ and $\left(y^{2}-a\right) \bmod p=0$ and $x \not \equiv y(\bmod p)$.

Let $f$ be a finite sequence of elements of $\mathbb{N}$ and let us consider $d$. One can check that $f(d)$ is natural.

The following propositions are true:

(16) Suppose $p>2$. Then there exists a finite sequence $f$ of elements of $\mathbb{N}$ such that

(i) len $f=\left(p-^{\prime} 1\right) \div 2$,

(ii) for every $d$ such that $d \in \operatorname{dom} f$ holds $\operatorname{gcd}(f(d), p)=1$,

(iii) for every $d$ such that $d \in \operatorname{dom} f$ holds $f(d)$ is quadratic residue $\bmod p$, and

(iv) for all $d, e$ such that $d, e \in \operatorname{dom} f$ and $d \neq e$ holds $f(d) \not \equiv f(e)(\bmod p)$.

(17) If $p>2$ and $a \operatorname{gcd} p=1$ and $a$ is quadratic residue $\bmod p$, then $a^{\left(p-{ }^{\prime} 1\right) \div 2} \bmod p=1$.

(18) If $p>2$ and $b \operatorname{gcd} p=1$ and $b$ is not quadratic residue $\bmod p$, then $b^{\left(p-{ }^{\prime} 1\right) \div 2} \bmod p=p-1$.

(19) If $p>2$ and $a \operatorname{gcd} p=1$ and $a$ is not quadratic residue $\bmod p$, then $a^{\left(p-^{\prime} 1\right) \div 2} \bmod p=p-1$.

(20) If $p>2$ and $a \operatorname{gcd} p=1$ and $a$ is quadratic residue $\bmod p$, then $\left(a^{(p-1) \div 2}-1\right) \bmod p=0$.

(21) If $p>2$ and $a \operatorname{gcd} p=1$ and $a$ is not quadratic residue $\bmod p$, then $\left(a^{(p-1) \div 2}+1\right) \bmod p=0$.

In the sequel $b$ is an integer.

We now state three propositions:

(22) Suppose $p>2$ and $a \operatorname{gcd} p=1$ and $b \operatorname{gcd} p=1$ and $a$ is quadratic residue $\bmod p$ and $b$ is quadratic residue $\bmod p$. Then $a \cdot b$ is quadratic residue $\bmod p$.

(23) Suppose $p>2$ and $a \operatorname{gcd} p=1$ and $b \operatorname{gcd} p=1$ and $a$ is quadratic residue $\bmod p$ and $b$ is not quadratic residue $\bmod p$. Then $a \cdot b$ is not quadratic residue $\bmod p$.

(24) Suppose $p>2$ and $a \operatorname{gcd} p=1$ and $b \operatorname{gcd} p=1$ and $a$ is not quadratic residue $\bmod p$ and $b$ is not quadratic residue $\bmod p$. Then $a \cdot b$ is quadratic residue $\bmod p$.

Let $a$ be an integer and let $p$ be a prime number. The functor $\left(\frac{a}{p}\right)$ yielding an integer is defined by: 
(Def. 3) $\left(\frac{a}{p}\right)=\left\{\begin{array}{l}1, \text { if } a \text { is quadratic residue } \bmod p, \\ -1, \text { otherwise. }\end{array}\right.$

One can prove the following propositions:

(25) $\left(\frac{a}{p}\right)=1$ or $\left(\frac{a}{p}\right)=-1$.

(26) If $a \operatorname{gcd} p=1$, then $\left(\frac{a^{2}}{p}\right)=1$.

(27) $\left(\frac{1}{p}\right)=1$.

(28) If $p>2$ and $a \operatorname{gcd} p=1$, then $\left(\frac{a}{p}\right) \equiv a^{\left(p-^{\prime} 1\right) \div 2}(\bmod p)$.

(29) If $p>2$ and $a \operatorname{gcd} p=1$ and $a \equiv b(\bmod p)$, then $\left(\frac{a}{p}\right)=\left(\frac{b}{p}\right)$.

(30) If $p>2$ and $a \operatorname{gcd} p=1$ and $b \operatorname{gcd} p=1$, then $\left(\frac{a \cdot b}{p}\right)=\left(\frac{a}{p}\right) \cdot\left(\frac{b}{p}\right)$.

(31) If for every $d$ such that $d \in \operatorname{dom} f^{\prime}$ holds $f^{\prime}(d)=1$ or $f^{\prime}(d)=-1$, then $\prod f^{\prime}=1$ or $\prod f^{\prime}=-1$.

In the sequel $m$ denotes an integer.

One can prove the following propositions:

(32) For all $g, f^{\prime}$ such that len $g=\operatorname{len} f^{\prime}$ and for every $d$ such that $d \in \operatorname{dom} g$ holds $g(d) \equiv f^{\prime}(d)(\bmod m)$ holds $\prod g \equiv \prod f^{\prime}(\bmod m)$.

(33) For all $g, f^{\prime}$ such that len $g=\operatorname{len} f^{\prime}$ and for every $d$ such that $d \in \operatorname{dom} g$ holds $g(d) \equiv-f^{\prime}(d)(\bmod m)$ holds $\prod g \equiv(-1)^{\operatorname{len} g} \cdot \prod f^{\prime}(\bmod m)$.

In the sequel $f$ denotes a finite sequence of elements of $\mathbb{N}$.

Next we state several propositions:

(34) Suppose $p>2$ and for every $d$ such that $d \in \operatorname{dom} f$ holds $\operatorname{gcd}(f(d), p)=$ 1 . Then there exists a finite sequence $f^{\prime}$ of elements of $\mathbb{Z}$ such that len $f^{\prime}=$ len $f$ and for every $d$ such that $d \in \operatorname{dom} f^{\prime}$ holds $f^{\prime}(d)=\left(\frac{f(d)}{p}\right)$ and $\left(\frac{\prod f}{p}\right)=\prod f^{\prime}$.

(35) If $p>2$ and $\operatorname{gcd}(d, p)=1$ and $\operatorname{gcd}(e, p)=1$, then $\left(\frac{d^{2} \cdot e}{p}\right)=\left(\frac{e}{p}\right)$.

(36) If $p>2$, then $\left(\frac{-1}{p}\right)=(-1)^{\left(p-{ }^{\prime} 1\right) \div 2}$.

(37) If $p>2$ and $p \bmod 4=1$, then -1 is quadratic residue $\bmod p$.

(38) If $p>2$ and $p \bmod 4=3$, then -1 is not quadratic residue $\bmod p$.

(39) Let $D$ be a non empty set, $g$ be a finite sequence of elements of $D$, and $i, j$ be natural numbers. Then $g$ is one-to-one if and only if $\operatorname{Swap}(g, i, j)$ is one-to-one.

(40) Let $g$ be a finite sequence of elements of $\mathbb{N}$. Suppose len $g=n$ and for every $d$ such that $d \in \operatorname{dom} g$ holds $g(d)>0$ and $g(d) \leq n$ and $g$ is one-to-one. Then $\operatorname{rng} g=\operatorname{Seg} n$.

In the sequel $a, m$ are natural numbers.

Next we state several propositions: 
(41) Let $g$ be a finite sequence of elements of $\mathbb{N}$. Suppose $p>2$ and $\operatorname{gcd}(a, p)=1$ and $g=a \cdot \operatorname{idseq}\left(\left(p--^{\prime} 1\right) \div 2\right)$ and $m=$

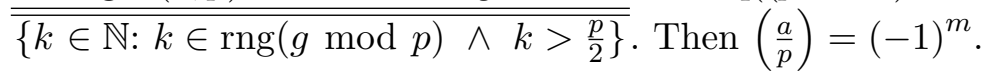

(42) If $p>2$, then $\left(\frac{2}{p}\right)=(-1)^{\left(p^{2}-^{\prime} 1\right) \div 8}$.

(43) If $p>2$ and if $p \bmod 8=1$ or $p \bmod 8=7$, then 2 is quadratic residue $\bmod p$.

(44) If $p>2$ and if $p \bmod 8=3$ or $p \bmod 8=5$, then 2 is not quadratic residue $\bmod p$.

(45) For all natural numbers $a, b$ such that $a \bmod 2=b \bmod 2$ holds $(-1)^{a}=$ $(-1)^{b}$.

In the sequel $g, g, h, k$ denote finite sequences of elements of $\mathbb{R}$.

Next we state two propositions:

(46) If len $g=\operatorname{len} h$ and len $g=\operatorname{len} k$, then $\left.g^{\frown} g-h\right\urcorner k=(g-h)^{\frown}(g-k)$.

(47) For every finite sequence $g$ of elements of $\mathbb{R}$ and for every real number $m$ holds $\sum$ (len $\left.g \mapsto m-g\right)=$ len $g \cdot m-\sum g$.

In the sequel $X$ denotes a finite set and $F$ denotes a finite sequence of elements of $2^{X}$.

Let us consider $X, F$. Then $\overline{\bar{F}}$ is a cardinal yielding finite sequence of elements of $\mathbb{N}$.

The following proposition is true

(48) Let $g$ be a finite sequence of elements of $2^{X}$. Suppose len $g=n$ and for all $d, e$ such that $d, e \in \operatorname{dom} g$ and $d \neq e$ holds $g(d)$ misses $g(e)$. Then $\overline{\overline{U \operatorname{rng} g}}=\sum \overline{\bar{g}}$.

In the sequel $q$ is a prime number.

The following three propositions are true:

(49) If $p>2$ and $q>2$ and $p \neq q$, then $\left(\frac{p}{q}\right) \cdot\left(\frac{q}{p}\right)=(-1)^{\left(\left(p-^{\prime} 1\right) \div 2\right) \cdot\left(\left(q-^{\prime} 1\right) \div 2\right)}$.

(50) If $p>2$ and $q>2$ and $p \neq q$ and $p \bmod 4=3$ and $q \bmod 4=3$, then $\left(\frac{p}{q}\right)=-\left(\frac{q}{p}\right)$.

(51) If $p>2$ and $q>2$ and $p \neq q$ and $p \bmod 4=1$ or $q \bmod 4=1$, then $\left(\frac{p}{q}\right)=\left(\frac{q}{p}\right)$.

\section{REFERENCES}

[1] Grzegorz Bancerek. Cardinal numbers. Formalized Mathematics, 1(2):377-382, 1990.

[2] Grzegorz Bancerek. The fundamental properties of natural numbers. Formalized Mathematics, 1(1):41-46, 1990.

[3] Grzegorz Bancerek. König's theorem. Formalized Mathematics, 1(3):589-593, 1990.

[4] Grzegorz Bancerek. The ordinal numbers. Formalized Mathematics, 1(1):91-96, 1990.

[5] Grzegorz Bancerek and Krzysztof Hryniewiecki. Segments of natural numbers and finite sequences. Formalized Mathematics, 1(1):107-114, 1990.

[6] Czesław Byliński. Binary operations. Formalized Mathematics, 1(1):175-180, 1990. 
[7] Czesław Byliński. Finite sequences and tuples of elements of a non-empty sets. Formalized Mathematics, 1(3):529-536, 1990.

[8] Czesław Byliński. Functions and their basic properties. Formalized Mathematics, 1(1):5565, 1990

[9] Czesław Byliński. Functions from a set to a set. Formalized Mathematics, 1(1):153-164, 1990.

[10] Czesław Byliński. The sum and product of finite sequences of real numbers. Formalized Mathematics, 1(4):661-668, 1990.

[11] Agata Darmochwał. Finite sets. Formalized Mathematics, 1(1):165-167, 1990.

[12] Zhang Dexin. Integer Theory. Science Publication, China, 1965.

[13] Yoshinori Fujisawa, Yasushi Fuwa, and Hidetaka Shimizu. Public-key cryptography and Pepin's test for the primality of Fermat numbers. Formalized Mathematics, 7(2):317-321, 1998.

[14] Hua Loo Keng. Introduction to Number Theory. Beijing Science Publication, China, 1957.

[15] Andrzej Kondracki. The Chinese Remainder Theorem. Formalized Mathematics, 6(4):573-577, 1997.

[16] Rafał Kwiatek. Factorial and Newton coefficients. Formalized Mathematics, 1(5):887-890, 1990.

[17] Rafał Kwiatek and Grzegorz Zwara. The divisibility of integers and integer relative primes. Formalized Mathematics, 1(5):829-832, 1990.

[18] Takaya Nishiyama and Yasuho Mizuhara. Binary arithmetics. Formalized Mathematics, 4(1):83-86, 1993.

[19] Dariusz Surowik. Cyclic groups and some of their properties - part I. Formalized Mathematics, 2(5):623-627, 1991.

[20] Michał J. Trybulec. Integers. Formalized Mathematics, 1(3):501-505, 1990.

[21] Zinaida Trybulec. Properties of subsets. Formalized Mathematics, 1(1):67-71, 1990.

[22] Edmund Woronowicz. Relations defined on sets. Formalized Mathematics, 1(1):181-186, 1990.

[23] Bo Zhang, Hiroshi Yamazaki, and Yatsuka Nakamura. Set sequences and monotone class. Formalized Mathematics, 13(4):435-441, 2005.

Received October 9, 2007 